\title{
A global small sunspot deficit at the base of the index anomalies of solar cycle 23
}

\author{
L. Lefèvre ${ }^{1}$ and F. Clette ${ }^{1}$
}

\begin{abstract}
Royal Observatory of Belgium (ROB), Circulaire 3, 1180 Bruxelles, Belgium
e-mail: [laure.lefevre; frederic.clette]@oma.be
\end{abstract}

Received 6 September 2011 / Accepted 16 November 2011

\begin{abstract}
Context. The variability of the 11-year cycle of solar activity on secular timescales is well established through the sunspot record, but it remains unpredictable. Indeed, the duration of the last solar cycle 23 was exceptionally long and took the solar physics community by surprise. The long minimum was marked by particularly low and often unprecedented levels of the international sunspot index $R_{\mathrm{i}}$ and most other solar indices. Earlier in the course of cycle 23, discrepancies appeared between several of those indices, raising a new issue: is there something wrong in the recent index values or is a real physical change occurring inside the Sun?

Aims. By exploiting detailed sunspot information, we look for independent evidence of a concrete and significant global change in sunspot properties appearing in the course of cycle 23.

Methods. To achieve this goal, we compared existing sunspot indices, such as the international sunspot number $\left(R_{\mathrm{i}}\right)$, and exploited the most complete information currently available on individual sunspots, obtained by merging two recent and complementary catalogs. Detailed statistics were obtained according to group type and spot size.

Results. We find that the Sun has shown an important deficit in small spots since the last activity maximum around 2000. While the number of large-scale spots remained largely unaffected, the occurrence rate of the smallest sunspots, and among them the ones with the shortest lifetimes, was more than halved during cycle 23. This explains the divergence between indices, weighted in favor of the largest active regions/magnetic structures, and sunspot counts that do not include such a weighting. It also confirms an actual intrinsic transition in the magnetic field generation inside the Sun, arising years before the exceptional activity minimum.

Conclusions. Our results thus reveal the potential of such detailed sunspot analyses for understanding and predicting future trends in the solar cycle. The change found here in the small individual sunspots suggests that solar and solar-terrestrial proxies should be redefined for the current state of the Sun, replacing the present ones. This scale-dependent change also provides support to dynamo models involving the coexistence of a deep and a superficial dynamo.
\end{abstract}

Key words. methods: data analysis - Sun: activity - sunspots - catalogs - Sun: dynamo

\section{Introduction}

During the 2007-2009 period, we experienced an unusually low solar minimum that the solar physics community was not able to predict (Pesnell 2008). Solar cycle (SC) 23 was exceptionally long, lasting about 12.4 years compared to 10.3 and 9.7 for SC21 and 22 (Emmert et al. 2010). During this last minimum, most solar indices also reached record-low levels: the international sunspot index $R_{\mathrm{i}}$ and the $10.7 \mathrm{~cm}$ radio flux $F_{10.7}$ (White et al. 2011), EUV fluxes (Solomon et al. 2010), and the total solar irradiance (Fröhlich 2009). Space weather indices, such as the ionospheric electron density characterized by the foF 2 ionosonde index or the TEC i.e. total electron content (Liu et al. 2011; Bergeot et al. 2011), also reached the lowest levels ever recorded. As these low values fall below the normal range consistently observed over several past SCs, this raises issues regarding the future evolution of solar activity and its output. In particular, a continuation of the above-mentionned, abnormally low flux levels would lead to unprecedented consequences on the Earth's environment.

To understand the underlying causes of this unexpected change, we need to identify possible precursor signs over SC23. So far, there have only been a few pieces of evidence: a decrease in the magnetic field strength of single sunspots during SC23 and 24 (Penn \& Livingston 2010), a slower migration of torsional oscillations (Howe et al. 2011), a faster meridional flow (Hathaway et al. 2010), a significant decrease in the Sun's polar magnetic field during the last minimum (Cliver \& Ling 2010; Wang et al. 2009; Smith \& Balogh 2008), a decrease in solar wind parameters (McComas 2008), and a change in the relative proportion of the small and large sunspot groups (Kilcik et al. 2011). In addition, two reference global solar indices $R_{\mathrm{i}}$ and $F_{10.7}$ started to show discrepancies around 2000 (Svalgaard \& Hudson 2010; Lukianova \& Mursula 2011), and $R_{\mathrm{i}}$ also started to disagree with other series like the Boulder sunspot number (Hathaway 2010). Those multiple discrepancies among solar indices raise a new issue: is there a flaw in some of the indices or are these distortions caused by a true physical change in the Sun?

This prompted us to investigate changes in the statistics of sunspots, in search of an explanation for the bias in the reference $R_{\mathrm{i}}$ index compared to other indices. Kilcik et al. (2011) find the first indication of a global deficit of small sunspot groups in SC23, compared to SC20 to 22. Here, we also focus on the sunspot record, but we use a new dataset providing fully detailed information, down to individual sunspots. In Sect. 2, we first validate the disagreements between indices by analyzing separately independent sunspot series from individual stations in the 
SIDC network, as well as other independent sunspot networks. In Sect. 3, we analyze the detailed information in a newly built sunspot catalog to reveal actual changes in the size distribution of sunspot groups and individual sunspots. Finally, in Sect. 4, we draw conclusions about the implications of those changes on the construction of long-term solar and geophysical proxies and on physical models of the solar dynamo.

\section{A disagreement between indices}

We start by investigating possible biases in the international sunspot index $R_{\mathrm{i}}$. This reference index is derived from a statistical average of sunspot counts, according to Wolf's formula $R_{Z}=10 N_{\mathrm{G}}+N_{\mathrm{S}}$ where $N_{\mathrm{G}}$ and $N_{\mathrm{S}}$ are the numbers of groups and spots, respectively (Wolf 1850). The index, based on 80 stations, is tied to the preceding $R_{Z}$ Zürich series by using the Locarno observatory (Specola Solare Ticinese) as pilot station (Clette 2007). Sergio Cortesi has been the main observer continuously since 1957 . We thus first verified that the $R_{\mathrm{i}}$ index is closely tracking the Locarno sunspot number during SC22 and 23. The RMS deviation over this period is $<3 \%$ while the peak to peak difference is always less than $5 \%$ and there is no significant trend. A flaw or change in the statistical $R_{\mathrm{i}}$ processing is thus excluded. Checking carefully the pilot station itself, we could not find any internal cause of a drift of the Locarno observations. There was a slight decrease in image quality (seeing) in 1998-2000, but it is much less than an earlier well-identified degradation in 1967-1973, which had no significant impact on the Zürich sunspot index. Possible modifications or degradations of the instrument were also investigated, as well as the eyesight of the principal observer against all the alternate observers of the Locarno station. No change could be found (Cortesi, priv. comm.).

Figure 1 compares Wolf numbers from individual stations inside both the $R_{\mathrm{i}}$-network and independent networks outside $R_{\mathrm{i}}$ over SC22 and 23. A significant difference appears after the maximum of SC23. While this difference between $R_{\mathrm{i}}$ and $F_{10.7}$ only becomes statistically significant in 2001, the divergence between indices seems progressive and starts already in the interval 1998-2000, as indicated by comparisons with the other series in Fig. 1. Here, we also show that, although Locarno forms the lower envelope of the network, most stations fall below the $F_{10.7}$ estimate, excluding an intrinsic bias present only in Locarno data, hence in $R_{\mathrm{i}}$. This collective change of independent index series strongly suggests that, instead of an artificial index bias in Locarno and $R_{\mathrm{i}}$, the explanation must be sought in a physical change on the Sun (visibility or actual number of sunspots).

\section{Exploration of the data: using detailed information}

The only way to explore this hypothesis is to use detailed information at the level of individual sunspots. For this purpose, we took advantage of a new comprehensive catalog that we built by merging two complementary catalogs that cover the 19862011 period: namely, the Debrecen Photoheliographic Data, DPD (Mezo 2005), listing all individual sunspots and the US Air Force SOON data ${ }^{1}$, USAF, listing numbered sunspot groups. The DPD catalog provides the position and area of each individual sunspot and of sunspot groups, distinguishing their umbra and penumbra, while the USAF catalog lists only sunspot groups

\footnotetext{
1 http://www .ngdc .noaa.gov/nndc/struts/results? op_ $\theta=e q \& v \_\theta=U S A F \_M W L \& t=102827 \& s=40 \& d=8 \& d=470 \& d=9$
}
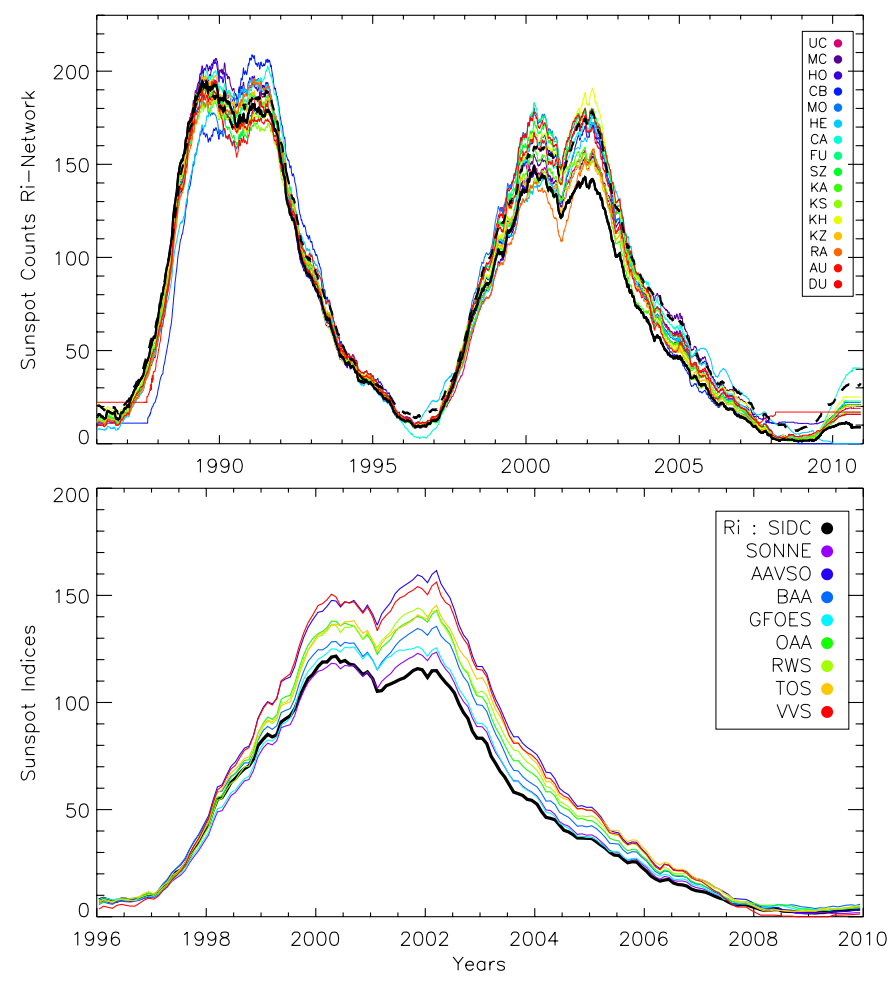

Fig. 1. Upper panel: sunspot numbers from 16 individual stations in the $R_{\mathrm{i}}$-Network (thin lines) and from the Locarno station (thick black line) compared with the $F_{10.7}$ flux (thick dashed line) transformed according to the $R_{2}$ monthly relation (Johnson 2011) $\left(F_{10.7}=67 \mathrm{sfu}\right)$. The station codes are listed in the legend. Lower panel: comparison between the $R_{\mathrm{i}}$ index (thick black) and 9 other independent sunspot networks (moving averages over one year). Abbreviations from the legend can be found on the SONNE website (http://www.vds-sonne.de/index.php? page $=$ gem $/$ res $/$ results. html).

but with important collective parameters like their McIntosh type (McIntosh 1990). We match each group entry of the DPD cata$\log$, i.e. a group and its individual spots, to an entry of the USAF catalog by comparing NOAA group numbers and positions, if necessary, correcting in the process a few inconsistencies in both catalogs (Lefèvre et al., in prep.). The success rate of this automatic matching is close to $97 \%$, thus requiring human intervention only for a few large complex active regions in order to complete the merging process.

In our sample, McIntosh types A and B correspond to small groups with small spots without penumbrae, while $\mathrm{C}$ to $\mathrm{F}$ are larger more developed groups containing at least one large spot with a substantial penumbra. Due to the interplay between seeing effects (blurred sunspot edges) and the intensity thresholding used by the Debrecen team (Gyori 1998), the splitting between the umbra $(\mathrm{U})$ and penumbra $(\mathrm{P})$ given in the DPD catalog is not trivial. Therefore, in addition to a criterion of size $\mathrm{U}+\mathrm{P} \leq 17 \mathrm{mil}-$ lionth of the solar hemisphere (msh), satisfied by $90 \%$ of the A and $\mathrm{B}$ groups, we used another criterion based on an $(\mathrm{U}+\mathrm{P}) / \mathrm{U}$ ratio $\leq 7$ in order to cleanly separate small spots without penumbra $(90 \%$ of $\mathrm{A}$ and $\mathrm{B}$ groups have $(\mathrm{U}+\mathrm{P}) / \mathrm{U} \leq 7)$. Thanks to the unique numbering of each sunspot group ${ }^{2}$, we can either simply sum up the instantaneous occurrence of all groups in each daily observation or include each group only once, at its maximum development (when its area is largest).

\footnotetext{
2 http://www. swpc.noaa.gov/ftpdir/forecasts/SRS/ README, \& /ftpdir/latest/SRS . txt
} 


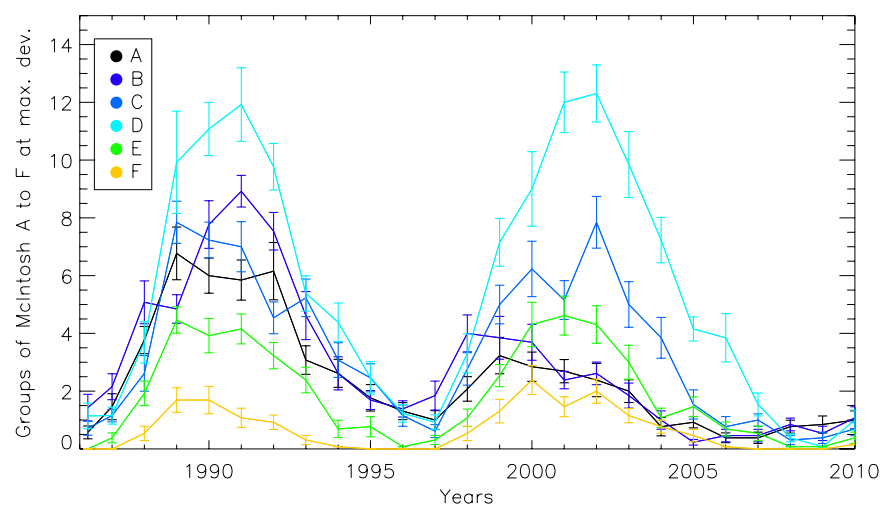

Fig. 2. Occurrence rates of groups of type A to F during SC22 and $\mathrm{SC} 23$. Error bars indicate the standard deviation over one year.

\subsection{Distribution of groups in the different Mclntosh types}

In the resulting catalog, we mostly use sunspot group information from the USAF/SOON input to count the rate of occurrence of each McIntosh type in groups at their maximum development. We compared two equivalent three-year time intervals centered on the maxima of cycles 22 [1989-1991] and 23 [2000-2002]. H-type groups correspond to a declining phase of evolution, so we excluded them from our analysis. We note that C-type groups, which were included among small groups by Kilcik et al. (2011), show only a minor deficit compared to A and B groups (cf. Sect. 3.3).

As shown in Fig. 2, for each group type, we find that the ratios of the average rates for the maximum of $\mathrm{SC} 22$ over the equivalent rates for the maximum of SC23 are $r_{\mathrm{A}}=2.4 \pm 0.5$, $r_{\mathrm{B}}=2.5 \pm 0.4, r_{\mathrm{C}}=1.2 \pm 0.2, r_{\mathrm{D}}=1.0 \pm 0.2, r_{\mathrm{E}}=1.0 \pm 0.2$, and $r_{\mathrm{F}}=0.8 \pm 0.3$. This means that $\mathrm{A}$ and $\mathrm{B}$ groups are two to three times less frequent during the maximum of SC23 compared to SC22. By contrast, D to F groups appear at almost the same rate. This anomaly appears to be specific to SC23, because SC22 shares the same overall properties with SC20 and 21 (Kilcik et al 2011). The difference thus seems to involve only small groups formed of small simple spots, while groups with larger spots seem unaffected. But does this change occur only at the level of the whole-group formation or at the level of all individual sunspots?

\subsection{Distribution of individual sunspots}

To clarify this, by now using all the details available only in the full USAF/DPD merged catalog, we counted all individual spots and extracted the number of small spots without penumbra inside the groups for types A to F during cycles 22 and 23 (Fig. 3). Here again, we observe a corresponding deficit of small spots in $\mathrm{SC} 23$ compared to SC22. For each group type, the ratios between maximum 22 and 23 are $r_{\mathrm{A}}=2.5 \pm 0.5, r_{\mathrm{B}}=3.7 \pm 0.7$, $r_{\mathrm{C}}=1.8 \pm 0.3, r_{\mathrm{D}}=1.2 \pm 0.2, r_{\mathrm{E}}=1.3 \pm 0.2$, and $r_{\mathrm{F}}=1.0 \pm 0.3$. A small spot deficit is thus found also in large $\mathrm{D}$ and $\mathrm{E}$ groups, suggesting that this shortage of small spots is not restricted to the smallest active regions. Without these detailed statistics on individual sunspots, Kilcik et al. (2011) wrongly attributed a decrease in the average sunspot area in large groups in SC23 to a larger proportion of small sunspots. To complement this analysis, Fig. 4 shows that the transition also occurs around 19982000 and only involves small spots inside all groups, while the number of larger spots shows no significant difference between the two cycles.

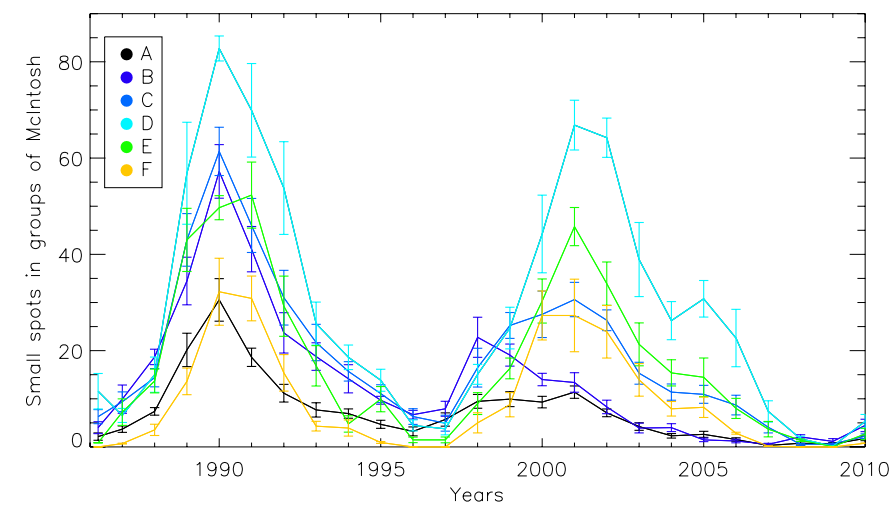

Fig. 3. Occurrence rates of small individual spots during SC22 and SC23 in groups of McIntosh type A to F. Small spots satisfy the criteria $(\mathrm{U}+\mathrm{P}) / \mathrm{U} \leq 7$ and $\mathrm{U}+\mathrm{P} \leq 17 \mathrm{msh}$.

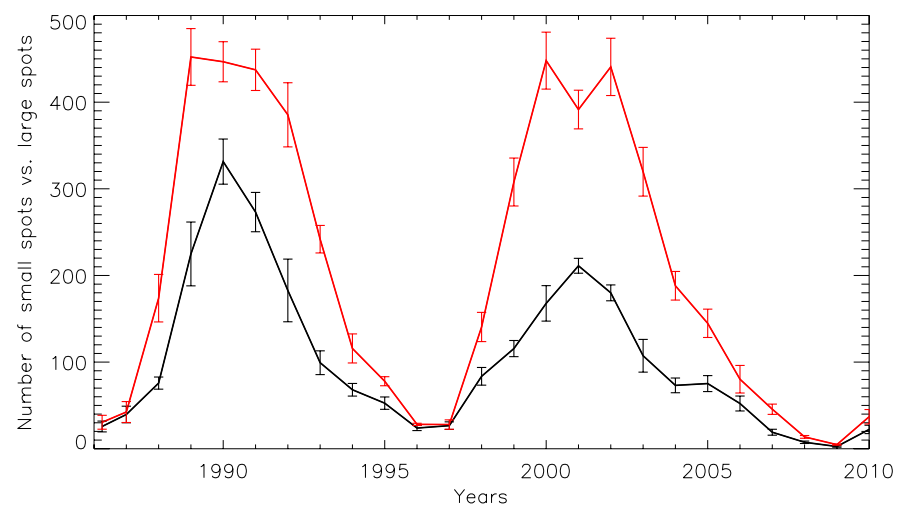

Fig. 4. Number of small (black line, $U+P \leq 17 \mathrm{msh}$ AND $U+P / U \leq 7$ ) and large individual spots (red line, $\mathrm{U}+\mathrm{P}>17 \mathrm{msh}$ OR $\mathrm{U}+\mathrm{P} / \mathrm{U}>7$ ) during cycles 22 and 23 . The monthly average sunspot counts were smoothed with a one-year running mean and are given with corresponding error bars.

This deficit in small spots emerging around 2000 should lead directly to a substantial decrease in the $R_{\mathrm{i}}$ index over the decline of SC23. Indeed, as by definition all spots contribute to $R_{\mathrm{i}}$ with an equal weight and small spots are the most abundant, the latter significantly contribute to the total $R_{\mathrm{i}}$ count. In contrast, most other indices, like $F_{10.7}$, based on total areas or fluxes, are strongly weighted in favor of high magnetic concentrations and are thus dominated by large active regions and spots. Therefore, changes affecting only small-scale magnetic elements should leave much weaker traces in those indices than they do in $R_{\mathrm{i}}$. Consequently, the relative decrease in $R_{\mathrm{i}}$ versus other solar indices can now be interpreted as a natural consequence of their different definitions, leading to a different sensitivity to the kind of change that is actually occurring on the Sun. Then, $R_{\mathrm{i}}$ simply emerges as the most sensitive index in this context, and the interindex divergence provides a diagnostic of a true solar evolution, without implying the failure of any index.

\subsection{Lifetime of sunspot groups}

Next to the spot counts, we also analyzed the distribution of sunspot groups lifetimes (Fig. 5). We find that there are fewer short-lifetime groups of types A, B, and a part of C in SC23 than in SC22. On the other hand, there is no significant difference in the distributions for D to F groups, even in the shortlifetime tails. For A and B types, the observed deficit is strongly 

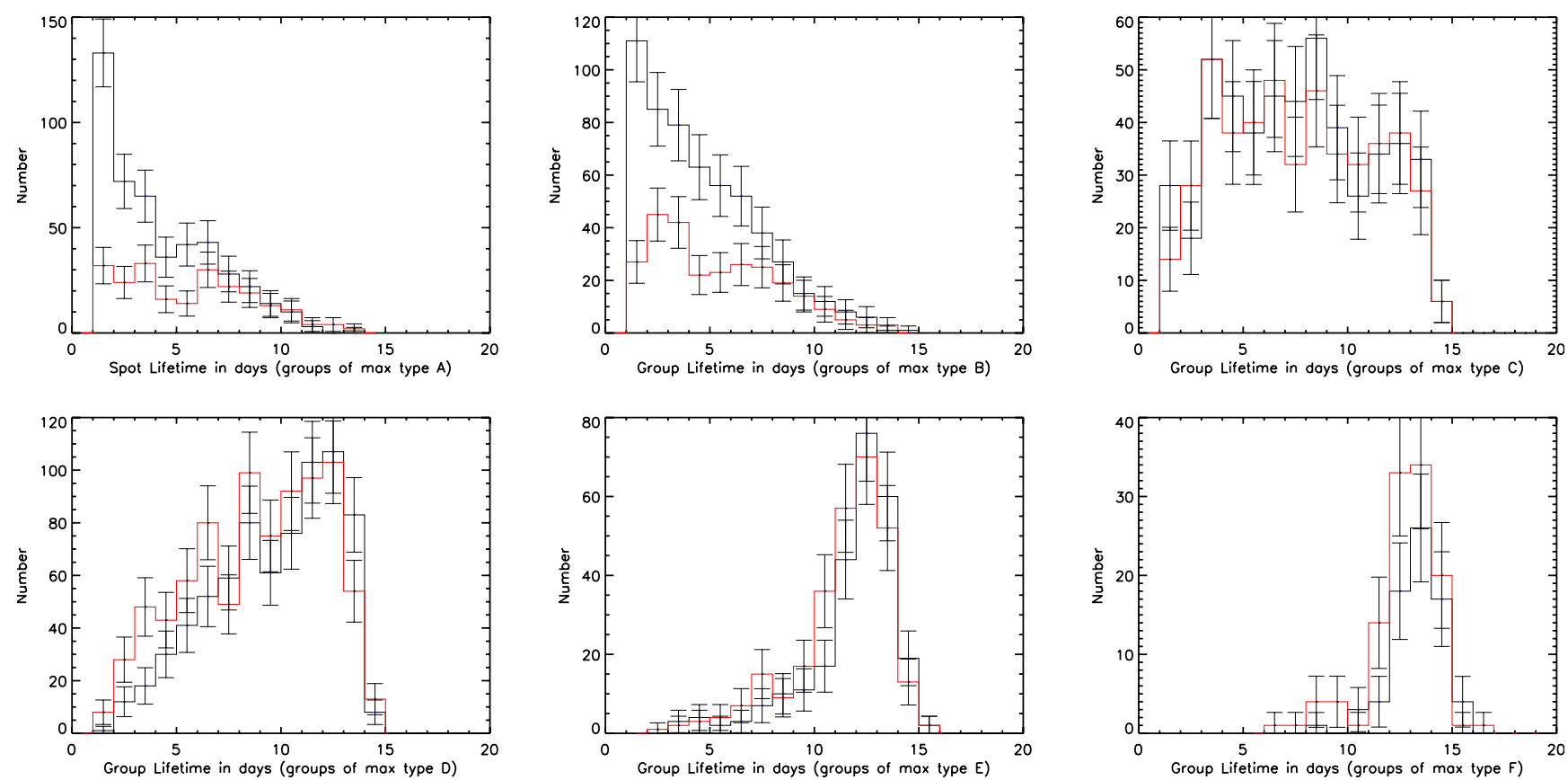

Fig. 5. Distribution of the group lifetimes for McIntosh types A to F in cycles 22 (black) and 23 (red). Errors bars give the $95 \%$ confidence level, assuming a binomial distribution of counts in each bin.

significant in cycle 23 and is largest in the lower lifetime range ( $<5$ days). We note that only $\mathrm{C}$-type group lifetimes have a wide and rather flat distribution (Fig. 5: upper right panel) intermediate between small $\mathrm{A}$ and $\mathrm{B}$ groups and large groups, suggesting a dual population. We can explain this by noting that the McIntosh C-class mixes groups with a small main penumbra, close to B type, and groups with a large main penumbra in a large decaying active region, close to the $\mathrm{H}$ type. This mixing will thus partly mask any trend in the short lifetimes for type $\mathrm{C}$. This shortage of short-lived sunspots thus complements the above indications of a numerical deficit in the smallest spots, because the latter have the shortest average lifetimes.

\section{Discussion and conclusions}

The global deficit of small spots found here sheds new light on the recent failure of the classical $F_{10.7}$-based proxies for the ionospheric foF2 and TEC indices that translate the atmospheric response to the solar UV and EUV irradiance (Liu et al. 2011; Bergeot et al. 2011). Our results support the conclusions drawn by Kilcik et al. (2011), but they also clarify and extend them to a global deficit of small sunspots in SC23. They strongly suggest that the Sun has switched to a new regime and, in particular, that this change is scale-dependent. This means that new proxy definitions will probably be required beyond a simple linear rescaling of the existing formulae. Those new sunspot-based proxies, based on post-2000 data, would probably allow a better reconstruction of SCs earlier than 1950, for which SC23 seems to be more representative.

Finally, this selective change which affects only the smallest magnetic elements, calls for a physical interpretation that goes beyond the scope of this paper. However, among the numerous theoretical models of solar magnetic field generation, some invoke the existence of two dynamos acting on two different scales: a deep global dynamo at the base of the convection zone and a local dynamo mechanism at work in the superficial layers on small scales (Schatten 2009). The scale-dependent change found here is compatible with this shallow dynamo model, which also happens to be one of the few that helped predict a late and weak SC24 (Pesnell 2008, Schatten 2005).

Acknowledgements. L.L. and F.C. wish to thank the Debrecen Heliophysical Observatory for advice on the use of their invaluable data, and in particular A. Ludmany, T. Baranyi, L. Gyori, and J. Murakozy for their help. This work has received funding from the European Community's Seventh Framework Program (FP7/2007-2013) under grant agreement No. 218816 (SOTERIA project, www. soteria-space.eu).

\section{References}

Bergeot et al. 2011, AGU Fall Meeting Abstracts, SA33B-1774 Chen, Y., Liu, L., \& Wan, W. 2011, J. Geophys. Res., 116, A4304

Clette, F., Berghmans, D., Vanlommel, P., et al. 2007, Adv. Space Res., 40, 919 Cliver, E.W., \& Ling, A.G. 2010, Sol. Phys., DOI:10.1007/s11207-010-9657-6 Emmert, J. T., Lean, J. L., \& Picone, J.M. 2010, Geophys Res. Lett., 37, 12102 Fröhlich, C. 2009, A\&A, 501, 27

Gyori, L., 1998, Sol. Phys., 180, 109

Hathaway, D. 2010, The Solar Cycle, Living Rev. Solar Phys., 7, 1

Hathaway, D., David, H., \& Rightmire L. 2010, Science, 327, 1350

Howe, R., Hill, F., Komm, R., et al. 2011, J. Phys., Conf. Ser., 271, 012074

Johnson, R.W. 2011, Astron. Space Sci., 332, 73

Kilcik, A., Yurchyshyn, V. B., Abramenko, V., et al. 2011, ApJ, 731, 30

Liu, L., Chen, Y., Le, H., et al. 2011, J. Geophys Res. 116, A4320

Lukianova, R., Mursula, K. 2011, J. Atm. Solar-Terrestrial Phys., 73, 235

McComas, D. J., Ebert, R. W., et al. 2008, Geophys. Res. Lett., 351, 18103

McIntosh, P. S. 1990, Sol. Phys., 125, 251

Mezo, G., \& Baranyi, T. 2005, Mem. Soc. Astron. Ital., 76, 1004

Neidig, D. et al. 1997, BAAS, 29, 897

Penn, M., \& Livingston, W. 2010, IAU 273, DOI:10.1017/S1743921311015122

Pesnell, W.D. 2008, Sol. Phys., 252, 209

Schatten, K. 2005, Geoph. Res. Lett., 32, 21106

Schatten, K. 2009, Sol. Phys., 255, 3

Smith, E. J., \& Balogh, A. 2008, Geophys. Res. Lett., 352, 22103

Solomon, S.C., Woods, T. N., Didkovsky, L. V., Emmert, J. T., \& Qian, L. 2010, Geoph. Res. Lett. 37, 16103

Svalgaard, L., \& Hudson, H.S. 2010, ASP Conf. Ser., 428, 325

Wang, Y.-M., Robbrecht, E., \& Sheeley, N.R. 2009, ApJ, 707, 1372

White, O. G., Kopp, M., Snow, K., Tapping., et al. 2011, Sol. Phys., DOI:10.1007/s11207-010-9680-7

Wolf, R. 1850, Astronomische Mitteilungen der Eidenössischen Sternwarte Zurich, 1, 27 